\title{
Evaluating the Curative Effect of Ultrasound- guided Sclerotherapy with Foam Lauromacrogol for Uterine Fibroids
}

\author{
Juan Gao, MS ${ }^{a}$, Yanggui Xie, $M S^{b, *}$, Bo Liang, MD ${ }^{b}$, Xiaoqin Qian, MD ${ }^{a}$ \\ ${ }^{a}$ Department of Ultrasound, Affiliated People's Hospital of Jiangsu University, Zhenjiang, China; ${ }^{b}$ Department of Ultrasonography, \\ Affiliated Hospital of Nantong University, Nantong, China \\ Received October 13, 2019; revision received March 24, 2020; accepted June 10, 2020
}

\begin{abstract}
Objective: To evaluate the curative effect of lauromacrogol foam sclerotherapy of uterine fibroids via transvaginal ultrasoundguided injection.

Methods: Thirty premenopausal women with a total of thirty-one uterine fibroids were recruited consecutively and underwent transvaginal 3D-Power Doppler Angiography (PDA) at 0, 1, 3, and 6 months. Written informed consent was obtained from all of the patients. The changes in the fibroid 3D volume and relationships between the fibroid location and blood supply were analyzed. The relief of clinical symptoms and improvement in health-related quality of life (HRQL) were evaluated using the uterine fibroid symptom and quality-of-life (UFS-QOL) questionnaire before and after treatment for 6 months; adverse events were also assessed.
\end{abstract}

Results: The mean baseline volumes $\pm \mathrm{SD}$ were $61.2 \pm 71.5 \mathrm{~cm}^{3}$ (range, $4.1 \mathrm{~cm}^{3}-340.4 \mathrm{~cm}^{3}$ ) for the dominant fibroid. The mean values of percentage reduction in fibroid volume at 1,3 and 6 months were $23.9 \%$ (range, 5.2\% $-42.1 \%$; $95 \%$ CI, 20.6\% $-27.3 \%$ ), $43.4 \%$ (range, $21.8 \%-67.4 \%$; 95\%CI, 39.0\% - 47.8\%), and 60.0\% (range, 31.8\% - 83.2\%; 95\%CI, 55.2\% - 64.9\%), respectively, and the reduction was correlated with the location and blood supply. Additionally, the blood flow to the fibroids was markedly decreased. The clinical symptoms were greatly alleviated or even completely resolved, and the HRQL was notably improved $(P<0.001)$. Mild abdominal pain, paleness, vomiting, and cold sweat were most commonly reported adverse effects.

Conclusion: Ultrasound-guided sclerotherapy of uterine fibroids with foam lauromacrogol is a simple, safe and useful method with a wide application prospect.

Key words: Uterine fibroid; Ultrasound-guided; Lauromacrogol; Sclerotherapy

Advanced Ultrasound in Diagnosis and Therapy 2021;01:032-038

DOI: 10.37015/AUDT.2021.190030

$\mathrm{U}$ terine fibroids are the most common benign tumor of the female reproductive tract, affecting $20-50 \%$ of women of reproductive age [1]. It is treated medically, surgically, or with other interventional methods. However, none of these therapies are a panacea.

Sclerotherapy involves the injection of a sclerosing agent for the targeted elimination of intracutaneous veins, resulting in the transformation of the veins into fibrous cords that cannot recanalize in the long term [2]. This technology has been used to treat rectal varices at the earliest, and the functional result is comparable to the surgical removal of a varicose vein [3]. We were, therefore, inspired to attempt lauromacrogol foam sclerotherapy of uterine fibroids via transvaginal

\footnotetext{
* Corresponding Author: Department of Ultrasonography, Affiliated Hospital of Nantong University, 20 Xisi Road, Nantong Jiangsu China.

e-mail:ntxieyg@163.com unrestricted use, distribution and reproduction in any medium provided that the original work is properly attributed.
} 
ultrasound-guided injection. It is a rapid and minimally invasive procedure, usually performed in an outpatient setting and does not require general anesthesia. Additionally, it offers the advantages of low patient discomfort, good tolerance, and rapid recovery, and the curative effect has been clinically verified [4].

Foam sclerosing agents are superior to the liquid ones since the former adheres easily to the vascular endothelium, thus increasing the time of adhesion and greatly decreasing the dosage of sclerosing agents, resulting in lower rates of complications [5]. The Tessari method for lauromacrogol foam sclerotherapy uses two $10-\mathrm{mL}$ syringes connected by a 3 -way stopcock connector; one of the syringes was filled with $10 \mathrm{~mL}$ air, while $5 \mathrm{~mL} 1 \%$ lauromacrogol (Shaanxi Tianyu Pharmaceutical Co., Ltd., China; approved by H20080445) was aspirated into the other syringe [6]. The two syringes were then rapidly and alternately pumped until the mixture turned into foam.

\section{Patients and Methods}

\section{Patients}

A group of 30 premenopausal women with a total of 31 uterine fibroids were consecutively recruited at the ambulatory clinic of the Department of Ultrasonic at the Affiliated Hospital of Nantong University, China and underwent ultrasound-guided injection of transvaginal lauromacrogol foam sclerotherapy between July 2014 and July 2015. This is a prospective study, and all of the uterine fibroids were diagnosed by ultrasound images and clinical symptoms of patients such as menstrual bleeding, pelvic pain, frequent urination and possible infertility. Criteria for case selection: (1) Most patients had only one uterine fibroid by the ultrasound diagnosis; (2) Patients with symptomatic uterine fibroids caused by the volume increase, menstrual extension or adjacent organs compression with symptoms such as frequency, constipation, etc; (3) Fast fibroid growth (rich blood supply) but not yet reaching indications for hysterectomy, or patients who do not wish to undergo a hysterectomy (which requires general anesthesia, while our method is local anesthesia) or those who voluntarily request interventional treatment; (4) Failure using traditional non-surgical treatment, myoma eliminating postoperative relapse, refusal of surgery or patients who are not suitable for surgical treatment; (5) Sclerotherapy was indicated 3-7 days after menstruation for patients whose bleeding time (BT, 1 - 4min), clotting time (CT, test tube method 5 - $11 \mathrm{~min}$; slide 1 - $5 \mathrm{~min}$ ), and hepatic and renal functions were within normal limits. Criteria for case exclusion: (1) Patients with severe underlying diseases; (2) People who are pregnant; (3) Women under the age of 18 or over the age of 60 . The ultrasound intervention room was equipped with a senior nurse and the necessary first aid equipment and medicine. The study protocol was approved by the local Institutional Review Board.

\section{Instruments and materials}

A medical ultrasound diagnostic instrument (MyLab Twice, Esaote, Italy) equipped with a transvaginal probe (EC1123; frequency, 5-8 MHz) and a specially matched puncture skeleton, which was routinely disinfected and individually packaged, was used in this study. First, all of the patients were administered perineum and vaginal disinfection and toweled in the bladder lithotomy position after emptying their bladders. Then, the transvaginal probe wrapped with sterile probe sets and coated with the special puncture guide frame was introduced into the patients' vaginas; the best puncturing path was chosen close to the posterior vaginal fornix in order to avoid injuring important organs such as the ovaries, bladder, and blood vessels. The puncture needle (Japan Baguang PTC needle, 19G) was then inserted via the special puncture guide frame into the sub-pseudocapsule where the fibroid was determined to be hypervascular according to its 3D-PDA 3D vessel glass-body mode image. Prior to the administration of foam sclerotherapy, local anesthesia ( $2 \mathrm{ml}$ of lidocaine hydrochloride), which can be visualized under ultrasound monitoring, was injected in order to ascertain the puncture site. The lauromacrogol foam was then injected under ultrasound guidance through the puncture needle. A ring of hyperechogenic mist flow resulting from rapid lauromacrogol foam diffusion (5-10 s) around the pseudocapsule was observed (Fig. 1). At the end of the treatment, the patients were instructed to stay in a sitting position for $1 \mathrm{~h}$ and were closely monitored. In case of postprocedural pain, an indomethacin suppository was administered. All of these procedures were performed by one of the investigators with 10 years of experience in ultrasound in gynecology.

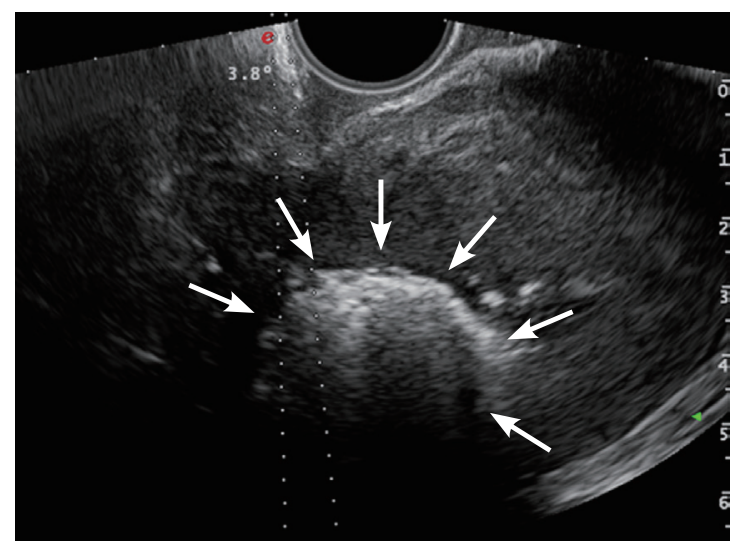

Figure 1 A ring of hyperechogenic mist flow (arrows) indicating rapid lauromacrogol foam sclerotherapy diffusion around the pseudocapsule. 
All of the ultrasound examinations were performed by another investigator using an ACCUVIX A30 Prestige system (Samsung Medison, Seoul, South Korea) with a V5-9 multifrequency transvaginal 3D probe (7-23 $\mathrm{MHz}$ ) before treatment and at 1,3, and 6 months after treatment, according to the predefined scanning protocol. The stored fibroid 3D volumes (Sonoview, ACCUVIX A30) were later analyzed by the same investigator using the VOCAL (Virtual Organ Computer-aided Analysis) program. In the end, the blood supply grades of all of the uterine fibroids were evaluated under the 3D-PDA 3D vessel glass-body mode using the Voluson E8 Expert ultrasound system (GE Healthcare, Milwaukee, WI, USA) with a RIC5-9-b multifrequency transvaginal 3D probe (6-22 MHz).

The following indices were evaluated at 1,3 , and 6 months after treatment: (1) Variations of fibroid 3D volume evaluated by 3D-PDA VOCAL. The mean percentage reduction in fibroid $=(1-$ Postoperative $3 \mathrm{D}$ volume/Preoperative 3D volume) $\times 100 \%$. (2) Variations of fibroid blood supply evaluated under the 3D-PDA $3 \mathrm{D}$ vessel glass-body mode. In accordance with Adler et al. [7] the distribution of the vessels supplying the fibroids was classified into four grades: grade 0/absent: no obvious blood flow signal visualized within the fibroid; grade $\mathrm{I} /$ minimal: one or two pixels containing flow (usually $<1 \mathrm{~mm}$ in diameter) visualized in the fibroid; moderate/grade II: a main vessel and/or several small vessels visualized in the area; marked/grade III: the presence of marked vascularity in the fibroids with four or more visualized vessels. (3) Relief of clinical symptoms and improvement of the health-related quality of life (HRQL) was evaluated using the UFS-QOL questionnaire [8].

All of the patients were followed up by transvaginal 3D-PDA at 1, 3, and 6 months after treatment, and the UFS-QOL was administered to all of the patients before treatment and 6 months posttreatment. The assessment endpoints included changes in the fibroid 3D volume and fibroid perfusion at 1,3 , and 6 months posttreatment as well as the UFS-QOL scores at 6 months posttreatment. In addition, adverse events during the follow-up period were recorded.

\section{Statistical analysis}

Descriptive statistical analysis was performed using IBM SPSS Statistics version 18.0 software. The descriptive data were expressed as the mean \pm standard deviation (SD). The Wilcoxon signed ranks test was performed to evaluate the variations in the fibroid 3D volume and blood supply before and after treatment. One-way ANOVA and Student's t-test were used to evaluate the variations in the fibroid $3 \mathrm{D}$ volume among different locations and blood supply grades. The variations in the fibroid blood supply among the groups of fibroids at different locations were compared using the Kruskal-Wallis and Mann-Whitney U tests. The ShapiroWilk test was used to evaluate the normal distribution of the continuous variables. The paired t-test was performed to evaluate the UFS-QOL score changes. All of the statistical tests were two-tailed and conducted with Type I error probability fixed at 0.05 .

\section{Results}

All of the 31 uterine fibroid sclerotherapy attempts were technically feasible (i.e., $100 \%$ technical success rate). The baseline characteristics of the patients with uterine fibroids are shown in Table 1. The mean age of the women was 40.3 years (range, 30-51). The number, size, and location of uterine fibroids were assessed for all of the patients; they included 16 intramural myomas, 8 submucosal myomas, and 7 subserosal myomas. Among 8 patients with menstrual bleeding, 5 patients had pelvic pain, and 3 patients had frequent urination. The fibroid 3D volumes ranged from $4.11 \mathrm{~cm} 3$ to $340.40 \mathrm{~cm} 3$. According to the blood flow, 12 of the fibroids were of grade III (Fig. 2A), 13 of grade II, and 6 of grade I.

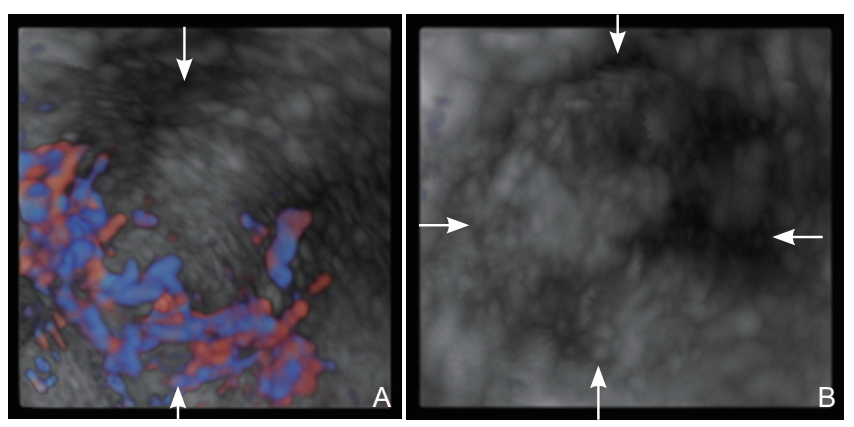

Figure 2 (A) The 3D-PDA 3D vessel glass-body mode shows the peripheral enlarged feeding vessels (arrows) in the fibroid before sclerotherapy, grade III; (B) The 3D-PDA 3D vessel glass-body mode shows no blood perfusion in the fibroid 6 months after sclerotherapy, grade 0 .

The mean values of percentage reduction in the fibroid $3 \mathrm{D}$ volumes were $23.9 \%, 43.4 \%$, and $60.0 \%$, respectively, at 1, 3, and 6 months (Fig. 3A and 4B), and all of these values were statistically significant compared to the pretreatment values $(P<0.001$; Table 2$)$. At 6 months posttreatment, only 2 fibroids of grade II, 6 of grade I, 23 of grade 0 (Fig. 2B) were present.

For factorial analysis, the patients were classified into two groups depending on whether the fibroid 3D volume was greater or lesser than $50 \mathrm{~cm} 3$; this cut-off volume point has been our empirical value over the past ten years. Group A contained 19 cases with relatively small fibroid 3D volumes $\left(<50 \mathrm{~cm}^{3}\right)$; group B contained 
12 cases with large fibroid $3 \mathrm{D}$ volumes $(\geqslant 50 \mathrm{~cm} 3)$. As shown in Table 4, the difference in the mean values of percentage reduction at 6 months posttreatment between group A and group B was not statistically significant
$(P=0.215)$. The changes in blood supply grades between the two groups also showed no statistically significant differences before $(P=0.857)$ and at 6 months after treatment $(P=0.589)$.

Table 1 Baseline characteristics of women with uterine fibroids

\begin{tabular}{|c|c|c|c|c|c|c|c|c|}
\hline \multirow{2}{*}{ Age $(Y)$} & \multicolumn{3}{|c|}{ Blood supply grades } & \multicolumn{3}{|c|}{ Type } & \multicolumn{2}{|c|}{ Volume $\left(\mathrm{cm}^{3}\right)$} \\
\hline & I & II & III & Submucosal & Intramural & Subserosal & $<50$ & $\geqslant 50$ \\
\hline$\leqslant 35$ & 1 & 2 & 4 & 1 & 5 & 1 & 7 & 0 \\
\hline $35-50$ & 4 & 10 & 8 & 6 & 10 & 6 & 11 & 11 \\
\hline$\geqslant 50$ & 1 & 1 & 0 & 1 & 1 & 0 & 1 & 1 \\
\hline
\end{tabular}
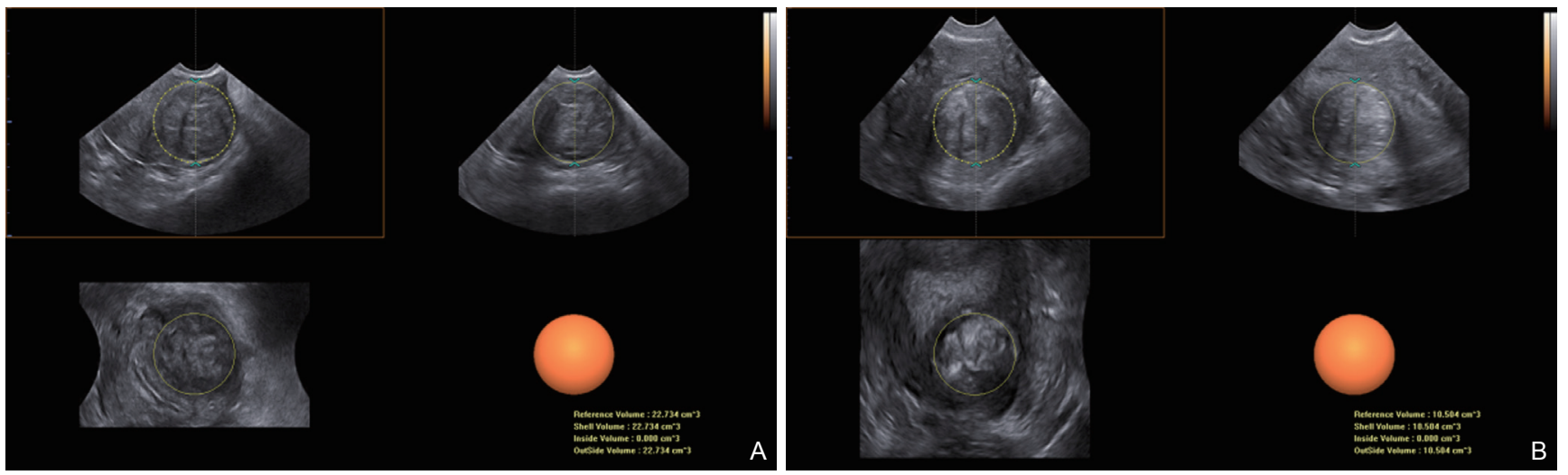

Figure 3 (A) Fibroid volume calculation using the 3D-PDA VOCAL software and spherical sampling before sclerotherapy; (B) Volume calculation of the same fibroid by using the VOCAL software and spherical sampling 6 months after sclerotherapy.

The fibroids were classified into three categoriesintramural, submucosal, or subserosal-based on the location. The intramural type was the most predominant subtype of fibroid identified $(n=16)$, and the submucosal type constituted the second largest group, accounting for 8 cases. Table 3 shows that the mean percentage reduction of the fibroid $3 \mathrm{D}$ volume was related to the location of the submucosal, intramural, and subserosal fibroids in descending order, and the percentage of fibroid 3D volume reduction at 6 months posttreatment in the intramural subtype was significantly different from that in the submucosal subtype, indicating a better prognostic trend in the submucosal subtype of fibroids. The differences in the fibroid blood supply grades among the fibroid groups at different locations before treatment had no statistical significance $(P=0.628)$. However, there were notable differences in the same at 6 months posttreatment $(P=0.003)$; the $\mathrm{p}$-values of the differences between the intramural-subserosal, subserosalsubmucosal, and submucosal-intramural groups of fibroids were $0.021,0.027$, and 0.490 , respectively.

As show in Figure 4, the percentage of fibroid 3D volume reduction at 6 months posttreatment was related to the blood supply grades III, II, and I in a descending order $(P=0.002)$. Grade III fibroids showed greater reduction of fibroid 3D volume compared with grade II and grade I fibroids. Comparison of the fibroid 3D volume reductions ratio of grades III, II, and I in pairs revealed that each of the pairs showed statistically significant differences, except for grades II and I $(P=0.061)$, indicating that the initial extent of fibroid vascularization might predict the decrease in fibroid volume after treatment.

The mean follow-up duration was 6 months. The UFS-QOL questionnaires were administered at each of the follow-ups, before and 6 months after treatment, and were assessed separately. The results show: 1) At baseline, the symptom severity scale scores were $60.82 \pm 92.39 ; 6$ months later, the symptom severity scale scores was $17.41 \pm 55.53$, from baseline to 6 months, the symptom severity scale scores ranged from 60.82 to 17.41 ; 2) At baseline, the HRQL scores was $44.57 \pm 34.64 ; 6$ months later, the HRQL scores was $93.62 \pm 13.51$, from baseline to 6 months, the HRQL scores ranged from 44.56 to 93.62 . The differences in the UFS-QOL scores pre and 6 months posttreatment 
were statistically significant $(P<0.001)$. Posttreatment complications occurred in seven of the patients. While five of the seven patients experienced mild abdominal pain $0.5 \mathrm{~h}$ after treatment, which was self-relieved in 1-3 h, two presented with paleness, vomiting, and cold sweat; they were asked to lie on their backs and were subcutaneously administered adrenaline hydrochloride $(0.5 \mathrm{mg})$ immediately. All of the patients were discharged upon observation for $1 \mathrm{~h}$ posttreatment. Oral prophylactic antibiotics, which are available over-the-counter at pharmacies, were suggested for 3-5 days posttreatment. Third generation cephalosporins were recommended, and the patient compliance was very good.

\section{Discussion}

In the present study, the fibroid 3D volumes were seen to have decreased progressively from 1 to 6 months posttreatment, indicating that the shrinkage of fibroids is a slow and gradual progress. Additionally, the 3D-PDA $3 \mathrm{D}$ vessel glass-body mode images revealed that the internal or surrounding blood flow of the fibroids decreased markedly in 6 months and the enlargement of the uterine fibroid was impossible. Therefore, the best curative effect observation window period was determined to be 6 months posttreatment.

In this study, we evaluated the 6-month impact of sclerotherapy in 30 women with 31 fibroids and investigated whether preoperative clinical and diagnostic features such as symptom presentation and fibroid size, vascularity, and location could predict successful therapy.

Our results (Table 3 ) indicate that the location was a useful predictor of treatment response. The intramural myomas and submucosal myomas in our cases responded better to treatment than did the subserosal myomas; additionally, the fibroids showed no remarkable differences in terms of the changes in fibroid blood supply, although they differed significantly in terms of the changes in fibroid 3D volumes. It may be postulated that this was due to the presence in such lesions of a vulnerable arterial supply, which was sensitive to oxygen, thus rendering the fibroids susceptible to sclerotherapy. This may be determined by the characteristic of the uterine myometrium vascular anatomy of the uterine blood preferring to flow to the uterus muscle lining [9]. Therefore, this can be used to select the most suitable uterine fibroid for sclerotherapy treatment.

Furthermore, the indication of lesion hypervascularity might be a strong and significant predictor of better prognosis in long-term survival analysis; this was intuitively confirmed in our study (Fig. 4). This finding was not unexpected, because the end point of treatment is a stasis of blood flow in the uterine arteries that supply feeding branches to the fibroids. In other words, vascularity has been shown to be an important factor in tumor growth, and the aim of lauromacrogol sclerotherapy is occlusion of the vessels of the perifibroid plexus and necrosis of the uterine fibroid cells; this directly leads to a decrease of tumor volume with the alleviation of clinical symptoms.

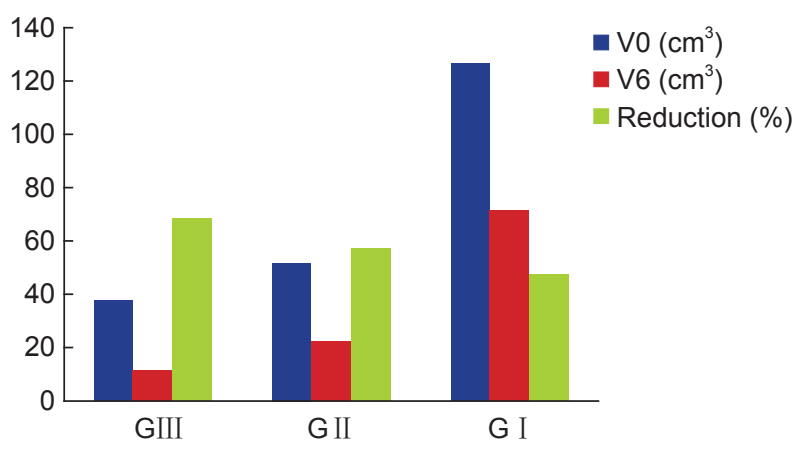

Figure 4 Changes in fibroid volume among groups of different grades before and 6 months after treatment. V0, fibroid volume before treatment; V6, fibroid volume at 6-month posttreatment; Reduction shows interclass comparison of the uterine fibroids volume before and 6 months after treatment.

The results presented in Table 4 show that our method has an equal curative effect regardless of the size of fibroid. Therefore, pretreatment fibroid size is not a useful predictor of sclerotherapy response.

The symptom severity and HRQL subscale scores of the UFS-QOL questionnaire indicate opposite outcomes, with higher symptom scores indicating greater clinical symptoms and higher HRQL subscale scores indicating better HRQL. Our data shows that all of the patients were symptomatic at baseline. At 6 months posttreatment, the symptom severity and UFS-QOL subscales were restored to normal values, and this normalization was sustained throughout the follow-up period (6 months). The results presented here indicate that the clinical symptoms of the patients were greatly alleviated and their HRQL improved with treatment. The improved symptoms did not recur during the follow-up.

It is noteworthy that the curative efficiency of sclerotherapy is consequently related to fibroid shrinkage and blood flow decrease. Therefore, a more rigorous assessment method is particularly important. The combination of 3D-ultrasound and power Doppler-3Dpower Doppler angiography (3D-PDA) - is a relatively new technique which can be used to assess the fibroid size and location as well as to depict uterine fibroid vascularity accurately owing to its advantages of noninvasiveness, low-cost, simple operation, easy acceptance by patients, and proven reproducibility $[10,11]$.

Fibroid volume was first measured by $2 \mathrm{D}$ 
ultrasonography, using the ellipsoid technique (volume $=$ length $\times$ width $\times$ depth $\times 0.523$; HWL method, for short). This method was initially applied to measure the urinary bladder, prostate, etc [12]. However, this method can cause measurement errors in most cases because of nonconformance to the physical contours of the organs. Three-dimensional ultrasonography, on the other hand, not only facilitates the volumetric study of organs and structures, but also provides a third image plane - the coronal plane - which allows more precise volumetric calculation, mainly of irregular shapes [13]. Although the measurements obtained by the rotational (VOCAL) and multiplanar methods have similar accuracy rates, the former has been shown to save time and is less affected by the inexperience of the operator [14]; its accuracy and repeatability have been proven by a considerable number of studies [11].

Table 2 Changes in fibroid volume and blood supply grades at $0,1,3$, and 6 months of treatment

\begin{tabular}{llll}
\hline Time $(\mathrm{M})$ & Volume $\left(\mathrm{cm}^{3}\right)$ & Reduction (\%) & Blood supply grades \\
\hline Average value & & & 96.66 \\
\hline 0 & $61.218 \pm 71.534$ & $23.958 \pm 9.22$ & $73.50^{\S}$ \\
\hline 1 & $48.091 \pm 61.827^{\dagger}$ & $43.418 \pm 11.99$ & $49.56^{\S}$ \\
\hline 6 & $37.247 \pm 51.727^{\dagger}$ & $60.041 \pm 13.14$ & $30.27^{\S}$ \\
\hline
\end{tabular}

${ }^{\dagger}$ Interclass comparison of the fibroid volume reduction ratio before treatment and at 1,3 , and 6 months posttreatment, $(P<0.001) ;{ }^{\S}$ Interclass comparison of and the blood supply before treatment and at 1,3 , and 6 months posttreatment; $P<0.001$.

Table 3 Changes in fibroid volume and blood supply before and 6 months after treatment

\begin{tabular}{lccccc}
\hline Fibroid location & $N$ & V0 $\left(\mathrm{cm}^{3}\right)$ & V6 $\left(\mathrm{cm}^{3}\right)$ & Reduction $(\%)$ & B0 Average value \\
\hline Submucosal & 8 & $46.57 \pm 28.20$ & $11.74 \pm 8.46$ & $74.79 \pm 5.40$ & 48.06 \\
Intramural & 16 & $53.43 \pm 64.99$ & $23.64 \pm 36.11$ & $60.09 \pm 7.93$ & 45.53 \\
Subserosal & 7 & $95.75 \pm 110.71$ & $56.21 \pm 66.62$ & $43.07 \pm 6.77^{\dagger}$ & $43.00^{\S}$ \\
\hline
\end{tabular}

N, number of fibroids; V0, fibroid 3D volume before treatment; V6, fibroid volume at 6 months posttreatment; B0, grade of fibroid blood supply before treatment; B6, grade of fibroid blood supply at 6 months posttreatment. ${ }^{\dagger}$ Interclass comparison of the fibroid volume reduction ratio before treatment and 6 months posttreatment $(P<0.001) ;{ }^{\S}$ Interclass comparison of the blood supply before and 6 months after treatment.

Table 4 Fibroid volume and blood supply changes in groups of different fibroid sizes

\begin{tabular}{llllll}
\hline Fibroid size $\left(\mathrm{cm}^{3}\right)$ & $N$ & V0 $\left(\mathrm{cm}^{3}\right)$ & V6 $\left(\mathrm{cm}^{3}\right)$ & Reduction $(\%)$ & B0 Average value \\
\hline $\mathrm{A}<50$ & 19 & $29.34 \pm 13.41$ & $11.18 \pm 6.68$ & $62.40 \pm 11.67$ & 16.26 \\
$\mathrm{~B} \geqslant 50$ & 12 & $111.69 \pm 95.53$ & $54.43 \pm 60.39$ & $56.31 \pm 14.94^{\dagger}$ & $15.58^{\S}$ \\
\hline
\end{tabular}

$\mathrm{N}$, number of fibroids; V0, fibroid 3D volume before treatment; V6, fibroid volume at 6 months posttreatment; B0, grade of fibroid blood supply before treatment; B6, grade of fibroid blood supply at 6 months posttreatment. ${ }^{\dagger}$ Interclass comparison of the fibroid volume reduction ratio before treatment and 6 months posttreatment $(P<0.001) ;{ }^{\S}$ Interclass comparison of the blood supply before and 6 months after treatment.

Recently, the pre and posttreatment variation of uterine fibroid vascularity has been evaluated by Color Doppler Flow Imaging (CDFI) and Contrast-enhanced Ultrasound (CEUS). Compared with 3D-PDA, CDFI presents a section of $2 \mathrm{D}$ color only and cannot show the entire blood supply of the fibroid; however, the 3D-PDA $3 \mathrm{D}$ vessel glass-body mode, which is mostly used to reconstruct the blood supply of tumors, can provide a clear, direct, and stereoscopic image that shows blood flow in a uterine fibroid as a spherical net structure and, in some instances, can reveal collateral flow not depicted by uterine artery arteriography [10]. While CEUS, which is an invasive method, is complicated, expensive, and is not readily accepted by patients, 3D-PDA offers a less expensive and more accessible modality for the evaluation of fibroid volume and vascularity, which can be used as a tool for pretreatment prediction, posttreatment evaluation, and guidance to access the puncture site [15].

Of course, there are many kinds of minimally 
invasive treatments for uterine fibroids, such as, laparoscopic myomectomy, uterine arterial embolization and high intensity focused ultrasound. However, all of them can cause damage to the patients to some degree. Briefly, laparoscopic hysteromyoma of the uterus can cause uterine rupture [16], uterine arterial embolization may cause premature ovarian failure [17], and high intensity focused ultrasound may cause bowel perforation, and damage to the adjacent bladder and large blood vessels because of the increasing heat [18]. Ultrasound-guided sclerotherapy of uterine fibroids with foam lauromacrogol is a simple, safe and useful method, so it has a wide prospect for application.

It should be emphasized that this is a new interventional method to treat uterine fibroid, and it has been investigated for very important potential clinical applications. However, this study was limited to a small group of patients and mainly analyzed the technical aspects, safety, and efficiency of treatment. Therefore, follow-up studies are needed to assess the long-term $(>2$ years) clinical outcomes.

\section{Acknowledgments}

We thank Youjia Wu and Zhiyong Shen for their help in preparing the final manuscript.

\section{Conflict of Interest}

The authors have no conflict of interest to declare.

\section{References}

[1] Solbiati L, Ahmed M, Cova L, Ierace T, Brioschi M, Goldberg SN. Small liver colorectal metastases treated with percutaneous radiofrequency ablation: local response rate and long-term survival with up to 10-year follow-up. Radiology 2012; 265: 958-68.

[2] Rabe E, Pannier F. Sclerotherapy of varicose veins with polidocanol based on the guidelines of the German Society of Phlebology. Dermatol Surg 2010; 36 Suppl 2: 968-75.

[3] Sato T, Yamazaki K, Akaike J, Toyota J, Karino Y, Ohmura T. Retrospective analysis of endoscopic injection sclerotherapy for rectal varices compared with band ligation. Clin Exp Gastroenterol 2010; 3: 159-63.

[4] Zhou X, Xie Y, Cui Q, Xu X, He M, Liang B. [Safety and efficacies of ultrasound-guided sclerotherapy of uterine fibroids with lauromacrogol]. Zhonghua Yi Xue Za Zhi 2014; 94: 2204-6.
[5] Ikponmwosa A, Abbott C, Graham A, Homer-Vanniasinkam S, Gough MJ. The impact of different concentrations of sodium tetradecyl sulphate and initial balloon denudation on endothelial cell loss and tunica media injury in a model of foam sclerotherapy. Eur $J$ Vasc Endovasc Surg 2010; 39: 366-71.

[6] Frullini A, Cavezzi A. Sclerosing foam in the treatment of varicose veins and telangiectases: history and analysis of safety and complications. Dermatol Surg 2002; 28: 11-5.

[7] Adler DD, Carson PL, Rubin JM, Quinn-Reid D. Doppler ultrasound color flow imaging in the study of breast cancer: preliminary findings. Ultrasound Med Biol 1990; 16: 553-9.

[8] Spies JB, Coyne K, Guaou Guaou N, Boyle D, Skyrnarz-Murphy K, Gonzalves SM. The UFS-QOL, a new disease-specific symptom and health-related quality of life questionnaire for leiomyomata. Obstet Gynecol 2002; 99: 290-300.

[9] Gomez-Jorge J, Keyoung A, Levy EB, Spies JB. Uterine artery anatomy relevant to uterine leiomyomata embolization. Cardiovasc Intervent Radiol 2003; 26: 522-7.

[10] Muniz CJ, Fleischer AC, Donnelly EF, Mazer MJ. Three-dimensional color Doppler sonography and uterine artery arteriography of fibroids: assessment of changes in vascularity before and after embolization. J Ultrasound Med 2002; 21: 129-33.

[11] Merce LT, Alcazar JL, Engels V, Troyano J, Bau S, Bajo JM. Endometrial volume and vascularity measurements by transvaginal three-dimensional ultrasonography and power Doppler angiography in stimulated and tumoral endometria: intraobserver reproducibility. Gynecol Oncol 2006; 100: 544-50.

[12] Hamper UM, Trapanotto V, DeJong MR, Sheth S, Caskey CI. Threedimensional US of the prostate: early experience. Radiology 1999; 212: 719-23.

[13] Riccabona M, Nelson TR, Pretorius DH. Three-dimensional ultrasound: accuracy of distance and volume measurements. Ultrasound Obstet Gynecol 1996; 7: 429-34.

[14] Alcázar JL, Prka M. Evaluation of two different methods for vascular sampling by three-dimensional power Doppler angiography in solid and cystic-solid adnexal masses. Ultrasound Obstet Gynecol 2009; 33: 349-54.

[15] Peng S, Xiong Y, Li K, He M, Deng Y, Chen L, et al. Clinical utility of a microbubble-enhancing contrast ("SonoVue") in treatment of uterine fibroids with high intensity focused ultrasound: a retrospective study. Eur J Radiol 2012; 81: 3832-8.

[16] Seinera P, Arisio R, Decko A, Farina C, Crana F. Laparoscopic myomectomy: indications, surgical technique and complications. Hum Reprod 1997; 12: 1927-30.

[17] Spies JB, Cooper JM, Worthington-Kirsch R, Lipman JC, Mills BB, Benenati JF. Outcome of uterine embolization and hysterectomy for leiomyomas: results of a multicenter study. Am J Obstet Gynecol 2004; 191: 22-31.

[18] Coakley FV, Foster BR, Farsad K, Hung AY, Wilder KJ, Amling $\mathrm{CL}$, et al. Pelvic applications of MR-guided high intensity focused ultrasound. Abdom Imaging 2013; 38: 1120-9. 